\title{
Extinction under nine food deprivation levels'
}

JOHN W. COTTON, University of California, Santa Barbara, Calif. 93106, WINFRED F. HILL, Northwestern University, Evanston, III. 60201, and MITRI E. SHANAB, California State College, Fresno, Calif. 93726

One-hundred-eighty Ss received 60 acquisition trials under $22 \mathrm{~h}$ food deprivation and 30 extinction trials with nine different food drive levels for different groups. A regular increase in extinction running speed with drive was observed early in extinction.

The present experiment provides a comparison of extinction under nine different food deprivation levels following acquisition under $22 \mathrm{~h}$ deprivation. On the basis of studies having a similar training drive level, using a large number of testing drive levels, and employing a nondiscrimination task (Horenstein, 1951 , and Kimble, 1951$)^{2}$, it seems appropriate to expect a sharp increase from 0 to $6 \mathrm{~h}$ of deprivation and smaller increases beyond that point.

\section{SUBJECTS AND APPARATUS}

One-hundred-eighty male albino rats (Sprague-Dawley strain) ranging in age from 74 to 88 days at the beginning of training served in this experiment. A 4-in. $\times 8 \frac{1}{2}$-in. $\times 4-\mathrm{ft}$ runway (Lewis \& Cotton, 1960) was used. A photocell placed just outside the start box started a clock when $S$ entered the runway proper; a second photocell stopped the clock when $S$ had gone $44 \frac{1}{2}$ in. beyond the first photocell. The running times obtained were converted to running speeds.

\section{PROCEDURE}

\section{Pretraining}

The Ss were placed on a $22-\mathrm{h}$ food-deprivation schedule for seven days, each $S$ receiving $10 \mathrm{~g}$ of ground lab chow $1 \mathrm{~h}$ after the time of day experimentation would begin for him. Each $S$ received $5 \mathrm{~min}$ of handling daily during this period and ate six $45-\mathrm{mg}$ lab chow pellets during that $5 \mathrm{~min}$ or directly after if they were not finished then.

Acquisition

Acquisition occurred on the eighth and ninth days, each $\mathrm{S}$ receiving 30 trials daily under $22-\mathrm{h}$ food deprivation. On each trial $\mathrm{S}$ was placed in the start box and allowed to run down the alley to the goal box. After $10 \mathrm{sec}$ in the goal box (or longer if required before $S$ consumed the one-pellet reward there), $S$ was placed in his home cage for $15 \mathrm{sec}$ before beginning another runway trial. Throughout the experiment an $S$ which did not complete a run within $120 \mathrm{sec}$ was placed in the goal box and his time recorded as $120 \mathrm{sec}$.

Extinction

The Ss were randomly assigned to nine groups of 20 for extinction. After the second acquisition session, members of the 0-, 1-, 3-, 6-, 18-, and 22-h groups were allowed to eat for $1 \mathrm{~h}$, so selected that a day later, at the time usually spent in running, they would have been food-deprived for the stated time. For example, an $S$ to be run at $3 \mathrm{pm}$ under 18-h deprivation would be fed from 8 to $9 \mathrm{pm}$ on the second day of acquisition. No Ss were run further until the second day after acquisition ended, at which time the 0-, 1-, 3-, 6-, 12-, 18-, and 22-h groups were finishing their second cycle under the new drive schedule and the 30 - and $46-\mathrm{h}$ groups were finishing a first cycle under their respective schedules. Thirty extinction trials, exactly like acquisition except that food was never present in the goal box, were conducted. ${ }^{3}$

\section{Acquisition}

\section{RESULTS}

The data for the 60 acquisition trials were segregated by groups and an analysis of variance conducted even though differential treatment had not yet occurred. Since the obtained $F$ was less than one, there is no evidence for differential performance attributable to an unusual group assignment.

\section{Extinction}

Figure 1 depicts the mean running speeds of each groun for Trials $1-5$ and Trials $1-30$ of extinction, as well as for Trials $1-60$ of acquisition. Extinction performance is substantially below the mean speed across all groups for the last five trials of acquisition (27.7 in. per sec).

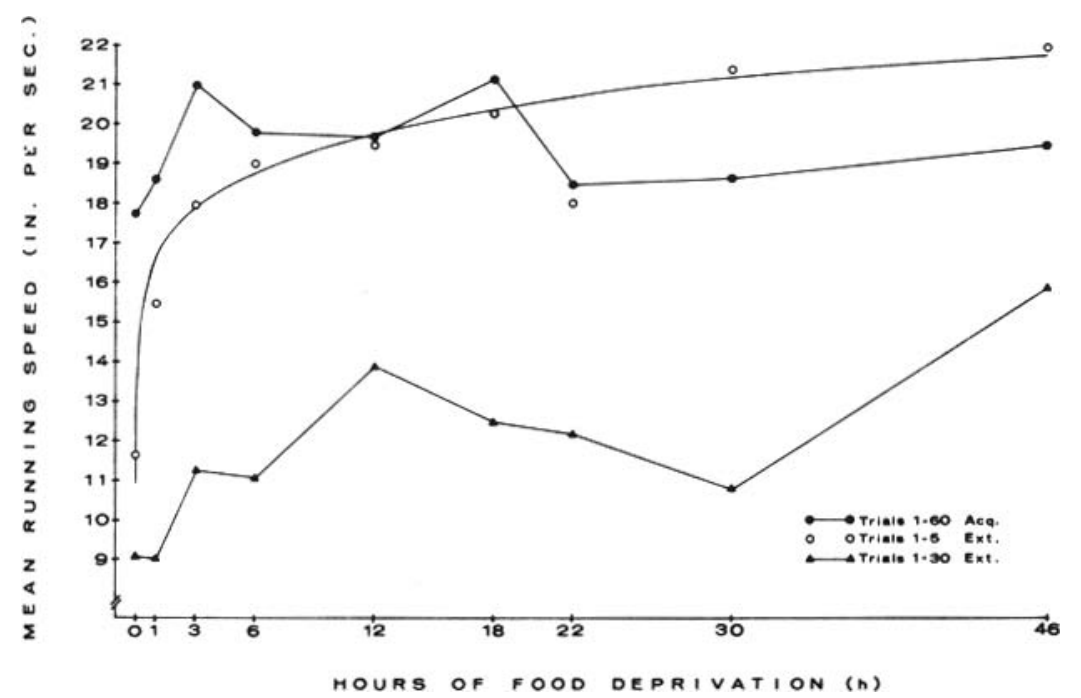

Fig. 1. A comparison of mean running speed in acquisition and two stages of extinction, as a function of extinction drive level (h). The smooth curve through individual data points conforms to the equation: $Y=10.94+5.76 h^{.17}$. 
Speeds for the first five trials of extinction are an almost monotonic increasing function of drive. The function for Trials 1-30 is clearly nonmonotonic for the observed data, though perhaps not for the population underlying the experiment. It is not clear whether the greater apparent effect of drive upon Trials 1-5 (as contrasted to Trials 1-30) of extinction is attributable to the greater level of performance early in extinction, or to drive stimulus generalization effects early in extinction, or both.

An analysis of variance of mean running speeds for the first five trials of extinction yielded a significant drive effect $(F=2.00, d f=8,171, p<.05)$. An orthogonal polynomials analysis of variance for unequally spaced intervals (Gaito, 1965) shows a significant linear effect $(F=9.12$, df $=1,171$, $p<.01$ ), but a test for nonlinearity shows no significant effects $(F=1.25$, df $=7,171, p>.05)$.

\section{DISCUSSION}

The general upward trend, with increased food drive, of running speeds during the first five trials of extinction confirms previous results. A steep slope of the behavior vs drive function for low drive levels is also customarily observed. Kimble's and Horenstein's findings of an inversion in the range from 0 to $1 \mathrm{~h}$ deprivation were not replicated, presumably because of our failure to use special satiation procedures. The most surprising result of the present study was an apparent drop in extinction running speed for the 22-h group, compared to the 18- and $30-\mathrm{h}$ groups. This may be a chance phenomenon, for we have no statistical evidence for a significant decline at that point; also the 22-h group was slow during acquisition.

Failure to find significant nonlinearity of drive effects upon the first five trials of extinction raises the question as to whether there is indeed a nonlinear trend as Horenstein's study, Kimble's study, and the mean values of the present study would suggest. So far as we know, there has been no previous statistical test of nonlinearity of drive effects. The question now arises whether any of the three experiments had sufficient power to lead us to expect a significant effect if one actually existed. This question has been investigated for the present study by determining the power which would exist if the true function were of the form $Y=10.94+5.76 \mathrm{~h} .17$ (where $\mathrm{Y}$ is mean running speed), a visually fitted function which is drawn through the data of Fig. 1. The further assumption has been made that the population variance is equal to 1.2786 , the error mean square obtained in the present experiment. These assumptions lead to a sum of squares for deviations from linearity of 10.1379 in the population. Use of Scheffé's Rule $1(1959$, p. 39 and p. 41$)$ gives a $\phi$ value of 1.00 , yielding a power of approximately .45 using the Pearson and Hartley charts (Scheffé, p. 444). If the number of cases per group had been 35 rather than 20, $\phi$ would have been 1.33 and the power about .72 . (An $\mathrm{N}$ of 35 with the same group means and variance as actually obtained in the present study would have yielded a barely significant deviation from linearity.) If the number of cases per group had been $80, \phi$ would have been 2.00 , and the power .99 .

Now consider power problems in the Horenstein and Kimble experiments. Each author concluded that analysis of variance was inappropriate to his data. However, in view of recent information on robustness and correlated measurements, it might have been legitimate to conduct an analysis of variance of repeated measurements for each experiment. Kimble had 60 observations per drive, and Horenstein had 100 , compared with the 100 (for the first five trials) of the present study. Kimble employed eight drives, Horenstein six, and the present experiment nine, which may affect the $\phi$ values from study to study. Neglecting that factor and assuming comparable drive changes in relation to variances, we can say that Kimble's use of the same Ss throughout would have to have produced variances only $60 / 100$ as large as with different Ss per drive in order to have the same power as in the present study. The same factors may have led to increased power in Horenstein's experiment.

We conclude that existing evidence for nonlinearity of drive effects is empirical rather than statistical, with Horenstein's experiment perhaps having provided the best information for a statistical answer to the question.

\section{REFERENCES}

GAITO, J. Unequal intervals and unequal $\mathrm{n}$ in trend analysis. Psychological Bulletin, 1965, 63, 125-127.

HORENSTEIN, B. J. Performance of conditioned responses as a function of strength of hunger drive. Journal of Comparative \& Physiological Psychology, 1951, 44, 210-224.

KIMBLE, G. A. Behavior strength as a function of the intensity of the hunger drive. Journal of Experimental Psychology, 1951, 41, 341-348. LEWIS, D. J., \& COTTON, J. W. Effect of runway size and drive strength on acquisition and extinction. Journal of Experimental Psychology, $1960,59,402-408$.

SCHEFFÉ, H. The analy sis of variance. New York: Wiley, 1959. NOTES

1. The experiment reported in this article was performed at Northwestern University with the support of National Science Foundation Grant NSF G-8706. Preparation of the manuscript was supported by a faculty research grant from the University of California, Santa Barbara. Appreciation is expressed to John Nielsen and Norman Spear for assistance.

2. Note that these two experiments measured reciprocal latency on rewarded trials as a function of deprivation at the time of testing, in contrast to the present investigation of extinction drive effects. Horenstein's study of extinction drive effects in the article cited used only four deprivation levels.

3. A subsequent extinction session introducing a new experimental variable is not described because of space limitations. 